\title{
Involvement of epithelial to mesenchymal transition in the development of pancreatic ductal adenocarcinoma
}

\author{
Kennichi Satoh $\cdot$ Shin Hamada $\cdot$ Tooru Shimosegawa
}

Received: 22 August 2014/Accepted: 29 August 2014/Published online: 14 September 2014

(C) Springer Japan 2014

\begin{abstract}
Pancreatic ductal adenocarcinoma (PDAC) is an intractable disease as a result of its rapid dissemination and resistance to conventional chemotherapy and radiotherapy. Surgical resection is the only curative therapy, but most of the tumors are unresectable at the time of diagnosis. The molecular mechanisms underlying the biological aggressiveness of this tumor type remain to be clarified. Epithelial to mesenchymal transition (EMT) is a developmental process that leads the phenotype shift from an epithelial morphology to a motile, fibroblast-like morphology. Recent studies showed that EMT is involved in the invasion and metastasis of many types of carcinomas including PDAC. In addition, PDAC cells with the EMT phenotype also exhibit chemoresistance and the cancer stem cell property. Various factors such as cytokines, growth factors, or transcriptional factors were found to promote the EMT program in PDAC cells. In this review, we summarize the current knowledge about the EMT in PDAC cells, focusing on the involvement of this process and its regulatory molecules including microRNA during the development of PDAC cells.
\end{abstract}

Part of this review was presented at The 4th International Forum of the 100th General Meeting of the Japanese Society of

Gastroenterology.

K. Satoh $(\bowtie)$

Division of Cancer Stem Cell, Miyagi Cancer Center Research Institute, 47-1 Nodayama, Medeshima-Shiote, Natori, Miyagi,

Japan

e-mail: ksatoh-gi@umin.ac.jp

S. Hamada $\cdot$ T. Shimosegawa

Division of Gastroenterology, Tohoku University Graduate

School of Medicine, Sendai, Miyagi, Japan
Keywords EMT - Pancreatic ductal adenocarcinoma . Cancer development

\section{Introduction}

Pancreatic ductal adenocarcinoma (PDAC) is one of the most malignant gastrointestinal tumors and is the fifth leading cause of cancer death in Japan [1]. Approximately, 32,330 people were diagnosed with PDAC and 28,017 people died from this disease in 2010 in Japan [1]. The 5-year relative survival rate, for all PDAC patients combined, was only $7 \%$ [2]. The reason for the lethal nature of PDAC is due to the difficulty of its early diagnosis and its rapid growth. Once PDAC becomes clinically evident, it is frequently accompanied by metastatic lesions at the time of diagnosis. In addition, this tumor usually shows resistance to conventional chemotherapy and radiation therapy. Therefore, new techniques for early diagnosis and/or a therapeutic strategy for advanced stage PDAC are required to improve the prognosis. Although recent molecular analyses of precursor lesions revealed an association between gene alterations and carcinogenesis [3], the pathogenic mechanisms that regulate the aggressive behavior of this cancer still remain to be clarified.

The process of epithelial to mesenchymal transition (EMT) was originally identified during specific stages of embryonic development in which cells migrate to and colonize different embryonic territories [4, 5]. Recently, EMT was shown to be a pivotal event during carcinoma progression and metastasis [5-8]. Tumor cells that undergo EMT show reduced intercellular adhesion with decreased expression of E-cadherin or translocation of $\beta$-catenin from cell membrane to nuclei, and obtain fibroblast-like properties with mesenchymal marker expression such as 
vimentin, fibronectin, and $\mathrm{N}$-cadherin. The transition of tumor cells from epithelial to mesenchymal features leads to increased migratory and invasive properties, and thus facilitates metastasis [5-8]. Moreover, accumulating evidence indicates the correlation between EMT and drug resistance or emergence of cancer stem cell (CSC) phenotypes [9-11]. In this review, we summarize the current knowledge about the involvement of EMT and its regulators (inducer and suppressor) in the biological aggressiveness of PDAC.

\section{EMT and invasion or metastasis of PDAC}

Among the various molecules associated with EMT, transforming growth factor (TGF) $\beta 1$ was the first identified cytokine that induced EMT in PDAC cells. Ellenrieder et al. [12] demonstrated that TGF- $\beta$ treatment led to the state of EMT in PDAC cell lines with a functional TGF- $\beta$ pathway (Panc-1, IMIM-PC-1, and COLO-357), characterized by a fibroblast-like morphology with upregulation of mesenchymal marker (vimentin) and downregulation of epithelial markers (cytokeratin-8 and -19), via cross talk with the Ras-MEK-ERK signaling pathway. The induction of EMT in PDAC cells by TGF- $\beta 1$ resulted in increased tumor cell migration, invasion, and scattering in vitro [12]. Subsequently, Yang et al. [13] found that vascular endothelial growth factor receptor-1 (VEGFR-1) promoted EMT in PDAC cells. Since the same group of authors had shown that the stimulation of PDAC cells by VEGFR-1 enhanced their migration and invasion in vitro [14], they investigated the underlying mechanism of this effect. The treatment with VEGFR-1 ligands (VEGF-A and VEGF-B) increased expression of vimentin and $\mathrm{N}$-cadherin, and decreased expression of E-cadherin with translocation of $\beta$ catenin from cell membrane to nucleus in the PDAC cell line L3.6pl [13]. In addition, activation of VEGFR enhanced expression of EMT-associated transcription factors, Snail, Twist, and Slug, indicating that VEGFR-1 mediated migration and invasion of PDAC cells through induction of EMT [13]. Then, EMT was found to be triggered by various secreted soluble factors such as bone morphogenetic protein (BMP) [15], Wnt [16], or macrophage migration inhibitory factor (MIF) [17], and was shown to be associated with invasion and metastasis of PDAC cells.

Hamada et al. [15] revealed that BMP4-treated Panc-1 cells exhibited loose cell contacts and scattered fibroblastoid appearance along with E-cadherin downregulation, vimentin upregulation, and enhanced cell migration, which are consistent with EMT phenotypes. During EMT acquisition by BMP4 treatment, homeobox gene MSX2 was markedly induced via the ERK and p38 mitogen-activated kinase (MAPK) pathways in Panc-1 cells. The characteristic of EMT disappeared when siRNA-based MSX2knockdown PDAC cells were treated with BMP4, indicating that MSX2 is indispensable for BMP4-induced EMT [15]. Next, our group investigated whether MSX2 itself could cause the EMT of PDAC cells. As endogenous MSX2 expression was low in BxPC3 and high in Panc-1 cells, several BxPC3-derived clones stably overexpressing MSX2 and Panc-1-derived clones stably expressing short hairpin RNA (shRNA) against MSX2 were generated [18]. MSX2-transfected BXPC3 cells showed loose cell junctions, scattered morphology, and fibroblast-like appearance compared with parental and empty vector (EV)-transfected cells. Meanwhile, parental Panc-1 and EV cells showed loose cell junctions and scattered morphology, while the MSX2-downregulated Panc-1 clones demonstrated a cobblestone-like phenotype. Reduced expression of E-cadherin and nuclear translocation of $\beta$-catenin were observed in MSX2-upregulated PDAC cells (MSX2-transfected BxPC3 cells and parental and EV-expressing Panc-1 cells) compared with MSX2-downregulated cells (EV-transfected BxPC 3 cells and MSX2 shRNA-introduced Panc-1 cells). These molecular changes in MSX2-expressing cells are identical to the EMT state. Consistent with the morphological and molecular changes, MSX2-expressing PDAC cells showed enhanced cell migration by wound-healing scratch assay and two-chamber assay, while downregulation of MSX2 in PDAC cells resulted in suppression of cell migration. This evidence clearly indicates that MSX2 itself plays a crucial role in the EMT of PDAC cells. In addition, MSX2-expressing PDAC cells exhibited enhanced metastasis to the liver while reduced expression of MSX2 in PDAC cells failed to form liver metastasis when orthotopic implantation was conducted in immunodeficient mice [18].

LIV-1, a member of new subfamily of zinc transporters, was shown to be involved in EMT of gastrula organizer cells in zebrafish [19] by regulating the nuclear translocation of Snail, which was demonstrated to be key regulator of EMT via repressing E-cadherin expression [20]. Unno et al. [21] revealed that LIV-1 was frequently expressed in PDAC tissues and its expression level was correlated with tumor size and lymphatic infiltration. To assess the functional role of this molecule in PDAC, they generated LIV1knockdown Panc-1. Decreased expression of LIV-1 in Panc-1 cells resulted in significant inhibition of cell motility in vitro and metastasis in vivo [21]. The fact that decreased nuclear expression of Snail and restored expression of E-cadherin in LIV-1 shRNA-expressing PDAC cells suggested that LIV-1 facilitates cellular migration and metastasis through the induction of EMT.

On the other hand, von Burstin et al. [22] selected a highly metastatic murine pancreatic cancer cell population by serial in vivo passaging of parental cells derived from 
TGF- $\alpha / \mathrm{p} 53^{+/-}$mice with low metastatic potential. They found that this in vivo selection of highly metastatic pancreatic cancer cells displayed the EMT phenotype accompanied by loss of E-cadherin expression and increased expression of mesenchymal genes including Twist- 1 and Snail. Silencing of E-cadherin by dominant-negative E-cadherin in parental cells altered the phenotype from epithelial to mesenchymal and increased the metastatic potential to the lung. In addition, highly metastatic PDAC cells from genetically engineered Kras-activated mice also showed the EMT phenotype with loss of E-cadherin expression and mesenchymal morphology, indicating the direct link between EMT and highly metastatic potential [22].

Özdemir et al. [23] generated transgenic mice with the ability to delete $\alpha \mathrm{SMA}+$ myofibroblasts in PDAC. They crossed $\mathrm{Ptf} 1 \mathrm{a}^{\text {cre/+}}$; LSL-Kras ${ }^{\text {G12D }}$; Tgfbr $2^{\text {flox/flox }}$ mice with aSMA-thymidine kinase (tk) transgenic mice to selectively target proliferating $\alpha \mathrm{SMA}+$ myofibroblast upon systemic ganciclovir administration. Depletion of $\alpha \mathrm{SMA}+$ myofibroblast starting at the precancerous stage and PDAC stage led to invasive and undifferentiated tumors with diminished animal survival, suggesting that the desmoplastic reaction may function as a protective response against carcinoma cell development rather than an oncogenic supportive role. Interestingly, PDAC cells that acquired invasive ability after depletion of myofibroblast exhibited phenotypic alteration to more EMT type with upregulation of EMT regulators Twist, Snail, and Slug.

These findings indicate that EMT can be induced by alteration of the microenvironment in addition to many factors derived from the cancer cell itself (autocrine) or stromal cells (paracrine), and that once the EMT program is processed, the ability of the PDAC cells to undergo invasion and metastasis is enhanced.

\section{EMT and chemoresistance}

Recent evidence indicates a close link between chemoresistance and the acquisition of the EMT phenotype in various carcinoma cells including PDAC [10]. PDAC cell lines (L3.6pl, BxPC3, CFPAC-1, and SU86.86) with high expression of E-cadherin and low expression of the mesenchymal marker Zeb-1 were demonstrated to be sensitive to chemotherapeutic agents [gemcitabine, 5-fluorouracil (5FU), and cisplatin] while other cell lines (Panc-1, Hs766T, AsPC-1, MIAPaCa2, and MPanc96) with the EMT state which express low level of E-cadherin and high level of Zeb-1 showed resistance to these drugs [24]. Downregulation of Zeb-1 in these PDAC cells with the EMT phenotype increased the expression of the epithelial marker and restored the drug sensitivity, suggesting that Zeb-1 and other regulators of EMT maintain the drug resistance of PDAC cells.

Meanwhile, Shah et al. [25] found that gemcitabineresistant PDAC (GR) cells, which were obtained by culturing in serially increasing concentrations of gemcitabine, underwent the morphologic changes consistent with EMT. These GR cells exhibited a 40- to 50-fold increase in resistance relative to parental cells with a reduction of E-cadherin and induction of vimentin expression [25]. The same group also revealed that Notch-2 and its ligand, Jagged-1, were upregulated in GR cells [26]. In addition, downregulation of Notch signaling in GR cells led to reversal of EMT along with reduced expression of vimentin, Zeb-1, Slug, Snail, and nuclear factor kB, suggesting that Notch signaling plays a crucial role in EMT induction [26]. Similarly, Güngör et al. [27] demonstrated the involvement of Notch signaling in chemoresistance associated with EMT in PDAC cells. They found that midkine (MK), a heparinbinding growth factor, was upregulated in gemcitabineinsensitive PDAC cells and its overexpression contributed to morphologic and molecular change consistent with EMT. Then, they identified an interaction between MK and Notch-2 receptor in PDAC cells, suggesting that MKNotch-2 interaction activates Notch signaling, promotes EMT, and increases chemoresistance [27].

The fact that chemoresistant PDAC cells possessed EMT characteristics raised the question whether induction of EMT would give rise to drug resistance. To address this issue, MSX2-expressing PDAC cells, which acquired the EMT phenotype as described above, were treated with 5-FU and gemcitabine [28]. Significant chemoresistance against 5-FU or gemcitabine was observed in MSX2-upregulated PDAC cells compared with empty vector-expressing control cells. By contrast, chemosensitization to 5-FU and gemcitabine was seen in MSX2-knockdown PDAC cells which showed more epithelial characteristics compared with control cells [28]. Salem et al. [29] also revealed the relationship between suppression of EMT and the attenuation of chemoresistance in PDAC cells. They showed that overexpressed Caveolin-1 (Cav-1) in PDAC cells resulted in increased membranous expression of E-cadherin and $\beta$ catenin, reduced cell migration and invasion, and chemosensitization to doxorubicin. In addition, Cav-1 induced downregulation of Snail expression and inactivation of EMT-inducible signal pathways such as AKT, MAPK, and TGF- $\beta$-Smad [29]. Collectively, acquisition of EMT is strongly associated with chemoresistance in PDAC cells.

\section{EMT and CSC property}

Carcinoma tissues consist of a heterogeneous cellular population containing a minor population of permanent 
proliferating cells and a major population of differentiated cells with limited proliferation potential. Among the permanent proliferating cells, so-called CSC, are considered to be responsible for the initiation, metastasis, chemoresistance, and recurrence of tumors [30]. CSCs are capable of self-renewing, differentiating into other cell types, and initiating tumors in immunodeficient mice. Cell surface markers including CD24, CD44, CD133, ESA, and their combinations have been employed to isolate CSCs from whole cancer cell populations [31, 32]. Li et al. [33] identified $\mathrm{CD}_{4} 4^{+} \mathrm{CD} 24^{+} \mathrm{ESA}^{+}$cells as pancreatic CSCs from PDAC tissues using fluorescence-activated cell sorting (FACS). They showed that implantation of as few as 500 of these $\mathrm{CD} 44^{+} \mathrm{CD} 24^{+} \mathrm{ESA}^{+}$cells could form tumors in NOD/SCID mice that recapitulated the architecture of original PDAC tissues. These $\mathrm{CD} 44^{+} \mathrm{CD} 24^{+} \mathrm{ESA}^{+}$cells had a 100 -fold tumorigenic potential in immunodeficient mice compared with these markers' negative PDAC cells [33]. Subsequently, Hermann et al. [34] demonstrated that $\mathrm{CD} 133^{+}$PDAC cells also had high tumor-forming ability and resistance to gemcitabine. Additionally, aldehyde dehydrogenase 1 (ALDH) [35] and c-Met [36] were identified as markers to isolate pancreatic CSCs.

Recent studies have revealed that CSC and EMT-type cells show similar characteristics such as chemoresistance and higher metastatic potential and share common molecular pathways including Notch and Wnt, suggesting the direct correlation between CSC property and EMT program [37]. In breast carcinoma cells, the CSC phenotype was found to be acquired by the forced expression of EMTinducing transcription factors, Snail and Twist [9]. Consistently, overexpression of Notch-1 enhanced not only the EMT property by upregulation of Zeb-1 but also the selfrenewal capacity with expression of CSC markers CD44 and ESA in the PDAC cell line AsPC-1 [38]. Forced expression of forkhead box protein M1 (Fox M1) also led to the EMT state by increased expression of Zeb-1, vimentin, and Snail2, and promoted the acquisition of the CSC phenotype with enhancement of self-renewal capacity as well as upregulation of CD44 and ESA in PDAC cells [39]. Similarly, induced expression of MSX2 in PDAC cells gave rise to the EMT phenotype and increased numbers of side population (SP) cells [28], which have been shown to be associated with CSCs [40, 41]. In addition, reversal of the EMT phenotype by silencing of Snail with shRNA introduction decreased the sphere formations, the expression of stem cell-related transcription factors Sox 2 and Oct4, and the tumorigenicity in vivo, in PDAC cells [42]. Furthermore, PDAC cells with the EMT program after depletion of $\alpha$ SMA myofibroblast exhibited increases in their capacity to form spheres in culture and numbers of $\mathrm{CD} 44^{+} \mathrm{CD} 133^{+}$cells, and the enhancement of tumorigenic potential in nude mice [23]. On the basis of these findings, promotion of EMT and deregulation of its regulators are likely to increase CSC properties and thus enhance aggressive behaviors of PDAC.

On the other hand, PDAC cells with the CSC phenotype displayed more enhanced migration ability than those otherwise when EMT was promoted by hypoxia [43]. Five PDAC cells were classified as less (CSC ${ }^{\text {low }}$ : BxPC3 and Capan-2) and highly aggressive CSC-like cell lines (CSC ${ }^{\text {high }}$ : MIAPaCa2, AsPC-1, and Capan-1) according to the degree of differentiation, spheroid-forming capacity, ALDH activity, and tumorigenicity in immunodeficient mice. Hypoxia altered the morphology of all cell lines from epithelial to mesenchymal phenotype while the effect was faster and stronger in $\mathrm{CSC}^{\text {high }}$ cells. In addition, although hypoxia enhanced the migration of all cell lines, velocity along with the percentage of migrating, polarized, and pseudopodia-forming cells was significantly higher in $\mathrm{CSC}^{\text {high }}$ cells [43]. These results suggested that only the CSC-like cells acquire high migratory potential and thus may be responsible for invasion and metastasis.

\section{MicroRNA and EMT}

MicroRNA (miR) is a small non-coding RNA which targets hundreds of mRNA, and its involvement in carcinoma progression is reported in various types of tumors including PDAC [44, 45]. MiR functions as either tumor initiator or suppressor depending on its downstream targets. It has been shown that all five members of the miR-200 family (miR-200a, miR-200b, miR-200c, miR-141, and miR-429) and miR-205 were downregulated in cells that had undergone EMT in response to TGF- $\beta$ and that enforced expression of the miR-200 family alone was sufficient to prevent TGF- $\beta$-induced EMT [46]. Using the target prediction program TargetScan, E-cadherin transcriptional repressor Zeb-1 and Zeb-2 were isolated as target for these miRs. Inhibition of these miRs (miR-200a, miR-200b, and miR-205) was found to induce EMT with upregulation of Zeb-1 and Zeb-2 in Madin Darby canine kidney (MDCK) epithelial cells. Consistently, loss of miR-200 family expression was detected in invasive breast cancer cell lines with mesenchymal phenotype. The miR-200 family was also downregulated in mesenchymal (sarcomatoid) breast carcinoma regions lacking E-cadherin expression, indicating that the miR-200 family regulates EMT by targeting Zeb-1 and Zeb-2 [46]. Li et al. [47] showed that three members of the miR-200 family (miR-200a, miR-200b, and $\mathrm{miR}-200 \mathrm{c}$ ) were downregulated in gemcitabine-resistant PDAC cells, which showed the EMT phenotype. Reexpression of the miR-200 family in PDAC cells with the EMT phenotype resulted in an increase in E-cadherin expression and decrease in Zeb-1 and vimentin expression, 
indicating the reversal of the EMT state [47]. Downregulation of miR-200b was also observed in the process of Notch-1-promoted EMT in AsPC-1 cells [38]. Re-expression of miR-200b in Notch-1-overexpressing AsPC-1 reversed the EMT phenotype, suggesting that miR-200b is involved in the EMT program partly through regulation of the Notch signaling pathway [38].

To explore the invasion-related miRs, comprehensive analysis comparing the miR expression profiles between PDAC and non-invasive intraductal papillary mucinous neoplasm of the pancreas (IPMN) was conducted. This experiment identified miR-126 [48] and miR-197 [49] as being significantly down- and upregulated miR in PDAC compared with IPMN, respectively. Interestingly, these miRs were associated with the EMT process in PDAC cells. Re-expression of miR-126 attenuated cellular migration and invasion along with induced expression of E-cadherin and reduced expression of mesenchymal marker N-cadherin in PDAC cells. ADAM9 (disintegrin and metalloproteinase domain-containing protein 9) was found to be a target of miR-126 and direct interaction between these molecules was confirmed by $3^{\prime}$ untranslated region ( $3^{\prime}$-UTR) assay. Knockdown of ADAM9 by siRNA in PDAC cells resulted in reduced cellular migration, invasion, and induction of E-cadherin [48]. On the other hand, expression of miR-197 by miR precursor introduction promoted invasion and migration in PDAC cells with decreased E-cadherin and increased vimentin expression that were in accordance with EMT induction. Reduced expression of p120 catenin, which was identified as the miR-197 target gene, in PDAC cells recapitulated EMT induction with increased migration, invasion, and expression of vimentin, and reduced E-cadherin expression [49].

miR-655 was shown to be EMT-suppressive miR by a cell-based reporter system employing a stable clone derived from Panc-1 transfected with a reporter construct containing a promoter sequence of E-cadherin in the $5^{\prime}$ upstream region of the ZsGreen1 reporter gene [50]. The ratio of fluorescence intensity of ZsGreen1 was measured using 470 synthetic double-strand RNAs mimicking human mature miRNAs, and miR-655 was identified as a novel EMT-suppressive miR in PDAC cells. Induction of E-cadherin expression and reduction of Zeb-1 were observed when miR-655 was introduced into Panc-1 cells. In addition, miR-655-expressing Panc-1 cells showed decreased cellular migration and invasion. The TargetScan database predicted Zeb-1 and TGFBR2 (type receptor of TGF- $\beta$ ) as the target of miR-655, and the direct link between them was validated by $3^{\prime}$-UTR assay. Consistently, overexpression of exogenous miR-655 could inhibit TGF- $\beta$-induced EMT by suppression of Zeb-1 and TGFBR2 expression in the PDAC cell line KP1N [50].

miRs were also shown to facilitate EMT in PDAC cells. Ouyang et al. [51] reported that the expression of miR-10b was increased in plasma of PDAC patients compared with normal controls. Interestingly, they revealed that miR-10b enhanced the stimulatory effects of epidermal growth factor (EGF) and TGF- $\beta 1$ on the induction of EMT in PDAC cells. The treatment of EGF together with TGF- $\beta 1$ induced invasion and migration along with reduced expression of E-cadherin and increased expression of $\mathrm{N}$-cadherin, vimentin, Snail, and Zeb-1 in PDAC cells (COLO-357 and
Fig. 1 EMT involvement during the development of pancreatic ductal adenocarcinoma (PDAC). EMT is induced by various factors including soluble secreted factors, transcription factors, microRNA, or microenvironment. Promotion of the EMT program enhances the ability of metastasis, chemoresistance, and characteristics of cancer stem cell phenotypes

Secreted soluble factors (TGF $\beta 1$, VEGF, BMP, EGF, etc).

EMT regulators (Snail, Slug, Zeb-1, MSX2, LIV-1, etc).

Notch signal.

Microenvironment condition (depletion of stroma, hypoxia).

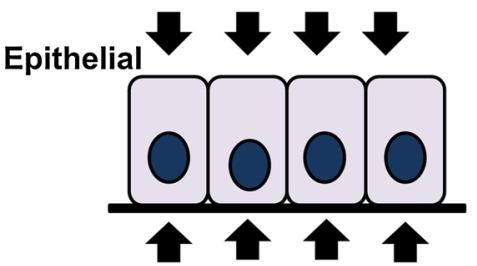

microRNA (miR-200 family, miR-126,-197, -655, -10b, etc).

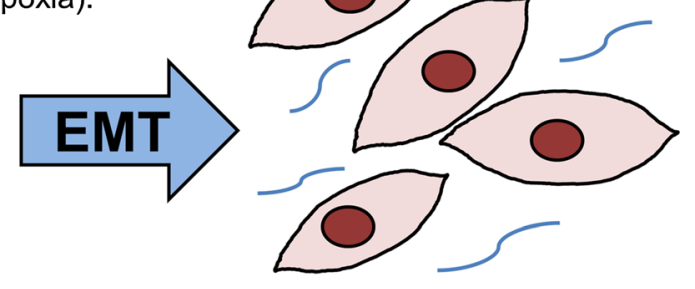

Invasion and metastasis Chemoresistance 1 Cancer stem cell property Development of PDAC 
Panc-1). This effect was significantly enhanced when miR$10 \mathrm{~b}$ precursor was transfected in these cells.

\section{Conclusion}

A number of studies demonstrate that EMT plays a crucial role not only in the process of invasion and metastasis but also in the acquisition of the CSC property as well as resistance to chemotherapy in PDAC cells. The roles of EMT in the development of PDAC are summarized in Fig. 1. EMT can be triggered by various factors such as cytokines, growth factors, transcription factors, and miRs in PDAC cells. Once the EMT program is activated by any of these triggers, PDAC cells enhance their malignant potential. Therefore, EMT and its regulators would be effective therapeutic targets for PDAC.

Conflict of interest The authors declare that they have no conflict of interest.

\section{References}

1. Matsuda A, Matsuda T, Shibata A, et al. A study of 25 populationbased cancer registries for the monitoring of cancer incidence in Japan (MCIJ) project. Jpn J Clin Oncol. 2013;44:388-96.

2. Monitoring of cancer incidence in Japan-survival 2003-2005 Report (Center for Cancer Control and Information Services, National Cancer Center, 2013). http://ganjoho.jp/professional/sta tistics/statistics.html.

3. Hong S, Park J, Hruban R, Goggins M. Molecular signatures of pancreatic cancer. Arch Pathol Lab Med. 2011;135:716-27.

4. Hay ED. An overview of epithelio-mesenchymal transformation. Acta Anat (Basel). 1995;154:8-20.

5. Thiery JP. Epithelial-mesenchymal transitions in development and pathologies. Curr Opin Cell Biol. 2003;15:740-6.

6. Thiery JP. Epithelial-mesenchymal transitions in tumor progression. Nat Rev Cancer. 2002;2:442-54.

7. Grünert $S$, Jechilinger M, Beug H. Diverse cellular and molecular mechanisms contribute to epithelial plasticity and metastasis. Nat Rev Mol Cell Biol. 2003;4:657-65.

8. Huber MA, Kraut N, Beug H. Molecular requirements for epithelial-mesenchymal transition during tumor progression. Curr Opin Cell Biol. 2005;17:548-58.

9. Mani SA, Guo W, Liao MJ, et al. The epithelial-mesenchymal transition generates cells with properties of stem cells. Cell. 2008;133:704-15.

10. Wang Z, Li Y, Ahmad A, et al. Pancreatic cancer: understanding and overcoming chemoresistance. Nat Rev Gastroenterol Hepatol. 2011;8:27-33.

11. Castellanos JA, Merchant NB, Nagathihalli NS. Emerging targets in pancreatic cancer: epithelial-mesenchymal transition and cancer stem cells. Onco Targets Ther. 2013;6:1261-7.

12. Ellenrieder V, Hendler SF, Boeck W, et al. Transforming growth factor beta1 treatment leads to an epithelial-mesenchymal transdifferentiation of pancreatic cancer cells requiring extracellular signal-regulated kinase 2 activation. Cancer Res. 2001;61:4222-8.

13. Yang AD, Camp ER, Fan F, et al. Vascular endothelial growth factor receptor-1 activation mediates epithelial to mesenchymal transition in human pancreatic carcinoma cells. Cancer Res. 2006;66:46-51.

14. Wey JS, Fan F, Gray MJ, et al. Vascular endothelial growth factor receptor-1 promotes migration and invasion in pancreatic carcinoma cell lines. Cancer. 2005;104:427-38.

15. Hamada S, Satoh K, Hirota M, et al. Bone morphogenetic protein 4 induces epithelial-mesenchymal transition through MSX2 induction on pancreatic cancer cell line. J Cell Physiol. 2007;213:768-74.

16. Bo H, Zhang S, Gao Li, et al. Upregulation of Wnt5a promotes epithelial-to-mesenchymal transition and metastasis of pancreatic cancer cells. BMC Cancer. 2013; doi: 10.1186/1471-2407-13-496.

17. Funamizu $\mathrm{N}, \mathrm{Hu} \mathrm{C}$, Lacy $\mathrm{C}$, et al. Macrophage migration inhibitory factor induces epithelial to mesenchymal transition, enhances tumor aggressiveness and predicts clinical outcome in resected pancreatic ductal adenocarcinoma. Int $\mathrm{J}$ Cancer. 2012;132:785-94.

18. Satoh K, Hamada S, Kimura K, et al. Up-regulation of MSX2 enhances the malignant phenotype and is associated with twist 1 expression in human pancreatic cancer cells. Am J Pathol. 2008;172:926-39.

19. Yamashita S, Miyagi C, Fukada T, et al. Zinc transporter LIVI controls epithelial-mesenchymal transition in zebrafish gastrula organizer. Nature. 2004;429:298-302.

20. Ikenouchi J, Matsuda M, Furuse M, et al. Regulation of tight junctions during the epithelium-mesenchyme transition: direct repression of the gene expression of claudins/occludin by Snail. J Cell Sci. 2003;116:1959-67.

21. Unno J, Satoh K, Hirota M, et al. LIV-1 enhances the aggressive phenotype through the induction of epithelial to mesenchymal transition in human pancreatic carcinoma cells. Int $\mathrm{J}$ Oncol. 2009;35:813-21.

22. von Burstin J, Eser S, Paul MC, et al. E-cadherin regulates metastasis of pancreatic cancer in vivo and is suppressed by a SNAIL/HDAC1/HDAC2 repressor complex. Gastroenterology. 2009;137:361-71.

23. Özdemir BC, Pentcheva-Hoang T, Carstens JL, et al. Depletion of carcinoma-associated fibroblasts and fibrosis induces immunosuppression and accelerates pancreas cancer with reduced survival. Cancer Cell. 2014;25:719-34.

24. Arumugam T, Ramachandran V, Fournier KF, et al. Epithelial to mesemchymal transition contributes to drug resistance in pancreatic cancer. Cancer Res. 2009;69:5820-8.

25. Shaah AN, Summy JM, Zhang J, et al. Development and characterization of gemcitabine-resistant pancreatic tumor cells. Ann Surg Oncol. 2007;14:3629-37.

26. Wang Z, Li Y, Kong D, et al. Acquisition of epithelial-mesenchymal transition phenotype of gemcitabine-resistant pancreatic cancer cells is linked with activation of Notch signaling pathway. Cancer Res. 2009;69:2400-7.

27. Güngör C, Zander H, Effenberger KE, et al. Notch signaling activated by replication stress-induced expression of midkine drives epithelial-mesenchymal transition and chemoresistance in pancreatic cancer. Cancer Res. 2011;71:5009-19.

28. Hamada S, Satoh K, Hirota M, et al. The homeobox gene MSX2 determines chemosensitivity of pancreatic cancer cells via the regulation of transporter gene ABCG2. J Cell Physiol. 2012;227:729-38.

29. Salem AF, Bonuccelli G, Bevilacqua G, et al. Caveolin-1 promotes pancreatic cancer cell differentiation and restores membranous E-cadherin via suppression of the epithelialmesenchymal transition. Cell Cycle. 2011;10:3692-700.

30. Reya T, Morrison S, Clarke M, et al. Stem cells, cancer, and cancer stem cells. Nature. 2001;414:105-11.

31. Clevers H. The cancer stem cell: premises, promises and challenges. Nat Med. 2011;17:313-9. 
32. Visvader JE, Lindeman GJ. Cancer stem cells: current status and evolving complexities. Cell Stem Cell. 2012;10:717-28.

33. Li C, Heidt DG, Dalerba P, et al. Identification of pancreatic cancer stem cells. Cancer Res. 2007;67:1030-7.

34. Hermann PC, Huber SL, Herrler T, et al. Distinct populations of cancer stem cells determine tumor growth and metastatic activity in human pancreatic cancer. Cell Stem Cell. 2007;1:313-23.

35. Kim MP, Fleming JB, Wang H, et al. ALDH activity selectively defines an enhanced tumor-initiating cell population relative to CD133 expression in human pancreatic adenocarcinoma. PLoS One. 2011;6:e20636.

36. Li C, Wu JJ, Hynes M, et al. c-Met is a marker of pancreatic cancer stem cells and therapeutic target. Gastroenterology. 2011;141:2218-27.

37. Li Y, Kong D, Ahmad A, et al. Pancreatic cancer stem cells: emerging target for designing novel therapy. Cancer Lett. 2013;338:94-100.

38. Bao B, Wang Z, Ali S, et al. Notch-1 induces epithelial-mesenchymal transition consistent with cancer stem cell phenotype in pancreatic cancer cells. Cancer Lett. 2011;307:26-36.

39. Bao B, Wang Z, Ali S, et al. Over-expression of FoxM1 leads to epithelial-mesenchymal transition and cancer stem cell phenotype in pancreatic cancer cells. J Cell Biochem. 2011;112:2296-306.

40. Dean M, Fojo T, Bates S. Tumor stem cells and drug resistance. Nat Rev Cancer. 2005;5:275-84.

41. Golebiewska A, Brons N, Bjerkvig R, et al. Critical appraisal of the side population assay in stem cell and cancer stem cell research. Cell Stem Cell. 2011;8:136-47.

42. Zhou W, Lv R, Qi W, et al. Snail contributes to the maintenance of stem cell-like phenotype cells in human pancreatic cancer. PLoS One. 2014;9:e87409.
43. Salnikov AV, Liu L, Platen M, et al. Hypoxia induces EMT in low and highly aggressive pancreatic tumor cells but only cells with cancer stem cell characteristics acquire pronounced migratory potential. PLoS One. 2012;7:e46391.

44. Szafranska AE, Davison TS, John J, et al. MicroRNA expression alterations are linked to tumorigenesis and non-neoplastic processes in pancreatic ductal adenocarcinoma. Oncogene. 2007;26:4442-52.

45. Yu J, Li A, Hong SM, et al. MicroRNA alterations of pancreatic intraepithelial neoplasias. Clin Cancer Res. 2012;18:700-12.

46. Gregory PA, Bert AG, Paterson EL, et al. The miR-200 family and miR-205 regulate epithelial to mesenchymal transition by targeting ZEB1 and SIP1. Nat Cell Biol. 2008;10:593-601.

47. Li Y, VandenBoom TG 2nd, Kong D, et al. Up-regulation of miR-200 and let-7 by natural agents leads to the reversal of epithelial-to-mesenchymal transition in gemcitabine-resistant pancreatic cancer cells. Cancer Res. 2009;69:6704-12.

48. Hamada S, Satoh K, Fujibuchi W, et al. MiR-126 acts as a tumor suppressor in pancreatic cancer cells via the regulation of ADM9. Mol Cancer Res. 2012;10:3-10.

49. Hamada S, Satoh K, Miura S, et al. MiR-197 induces epithelialmesenchymal transition in pancreatic cancer cells by targeting p120 catenin. J Cell Physiol. 2013;228:1255-63.

50. Harazono $\mathrm{Y}$, Muramatsu T, Endo $\mathrm{H}$, et al. miR-655 is an EMTsuppressive microRNA targeting ZEB1 and TGFBR2. PLoS One. 2013;8:e62757.

51. Ouyang H, Gore J, Deitz S, et al. microRNA-10b enhances pancreatic cancer cell invasion by suppressing TIP30 expression and promoting EGF and TGF-b actions. Oncogene. 2013; doi:10. 1038/onc.2013.405. 